\title{
Partir à l'étranger pour y ouvrir une entreprise de photos de " pré-mariage ». Le cas des acteurs chinois à Phuket
}

Starting a "Pre-Wedding" Photo Business Abroad. The Case of Chinese

Stakeholders in Phuket

\section{Meng Li}

\section{OpenEdition \\ Journals}

Édition électronique

URL : https://journals.openedition.org/tourisme/3520

DOI : $10.4000 /$ tourisme.3520

ISSN : 2492-7503

Éditeur

Association Mondes du tourisme

\section{Référence électronique}

Meng Li, «Partir à l'étranger pour y ouvrir une entreprise de photos de «pré-mariage ». Le cas des acteurs chinois à Phuket », Mondes du Tourisme [En ligne], 19 | 2021, mis en ligne le 15 septembre 2021, consulté le 17 septembre 2021. URL : http://journals.openedition.org/tourisme/3520 ; DOI https://doi.org/10.4000/tourisme.3520

Ce document a été généré automatiquement le 17 septembre 2021.

\section{cc)}

Mondes du tourisme est mis à disposition selon les termes de la licence Creative Commons Attribution - Pas d'Utilisation Commerciale - Pas de Modification 4.0 International. 


\title{
Partir à l'étranger pour y ouvrir une entreprise de photos de " pré- mariage ». Le cas des acteurs chinois à Phuket
}

\author{
Starting a "Pre-Wedding" Photo Business Abroad. The Case of Chinese \\ Stakeholders in Phuket
}

Meng Li

\section{Introduction}

1 Si le tourisme international débute en Thaïlande au tournant des années 1960, une nouvelle phase de son histoire semble actuellement se dérouler, avec l'accroissement des flux en provenance de la République populaire de Chine (Dai et al., 2017). Le phénomène est de plus en plus documenté par les chercheurs chinois, qui se concentrent principalement depuis plusieurs années sur la segmentation de ce « marché » (Untong, 2015). Très peu de textes s'intéressent en revanche aux acteurs touristiques en présence, qui prennent en charge le déplacement et l'accueil des touristes chinois. Cela sera l'objet de cet article, qui se penche sur le cas spécifique des opérateurs chinois venus en Thaïlande pour y tenter leur chance. Nous étudierons plus en détail le cas de Phuket, l'une des principales îles touristiques au sud du royaume.

2 Au-delà de l'ensemble des acteurs touristiques (soit « l'ensemble des agents (individus, groupes de personnes, organisations) [touristiques] susceptibles d'avoir, directement ou indirectement, une action sur les territoires ", Géoconfluences, 2020), notre intérêt se portera sur certains entrepreneurs, qui se sont spécialisés dans un secteur en développement, celui des photographies de "pré-mariage », à mi-chemin entre le voyage de noces et un séjour à thème. Alors que ces voyages étaient jusqu'alors principalement réalisés sur l'île de Hainan (la province la plus méridionale de Chine, au 
sud de Canton et de Hong Kong), on assiste depuis peu à une réorientation des flux vers les îles touristiques internationales d'Asie du Sud-Est. À partir d'analyses réalisées à Kamal, dans l'ouest de l'île de Phuket, notre objectif sera d'analyser la constitution d'un système d'acteurs touristiques, qui organise localement ce nouveau phénomène. Comme nous le verrons, certains entrepreneurs, basés autrefois en Chine, déménagent désormais à Phuket, afin de profiter de ce nouveau marché et de ses opportunités économiques. Nous tenterons donc de répondre aux questions suivantes : qui sont ces petits entrepreneurs venus de Chine ? Comment ont-ils fait pour accéder à ce marché et pour l'exploiter au sein de l'île? Comment s'organisent-ils actuellement, dans un contexte où leurs activités sont parfois illégales? Quelles sont, enfin, leurs interrelations avec les autres opérateurs locaux, ceux issus de la diaspora chinoise, les Thaillandais, mais aussi les autres entrepreneurs comme eux immigrants?

Pour ce faire, l'article est structuré en trois grandes parties. La première présente l'évolution du tourisme chinois en Thaïlande, en soulignant l'émergence et l'accroissement de sa part individuelle. Pour répondre à la demande grandissante de prise de photos de "pré-mariage ", des professionnels chinois viennent s'installer à Phuket. Nous en avons interrogé certains, selon des modalités qui seront précisées. L'objet de la deuxième partie est dès lors d'analyser comment s'organise cette immigration et son installation sur place, selon le profil de ces opérateurs. L'exemple de Kamala, à l'ouest de Phuket, sera privilégié. Enfin, la troisième partie explore plus spécifiquement la réorganisation des employés non chinois pour répondre aux besoins de la clientèle chinoise individuelle. La hiérarchie du travail peut s'en trouver réorganisée.

\section{Des voyagistes chinois qui s'installent à Phuket pour y travailler, sur le marché du tourisme international chinois individuel}

\subsection{Le tourisme international chinois en Thaïlande : I'importance croissante du tourisme individuel}

4 Apparu grâce au développement économique engagé depuis 1978, le tourisme international chinois est longtemps resté, jusqu'au début des années 2000 au moins, cantonné à des destinations proches. Les pays visités alors, majoritairement en Asie du Sud-Est, sont depuis devenus les principaux espaces récepteurs (Arlt, 2006). En Thaïlande, en particulier, la part des arrivées chinoises dans le volume international du pays augmente : en 2018, les Chinois représentaient $27 \%$ des touristes internationaux présents dans le royaume de Thaïlande, contre $10 \%$ pour les Européens et $3 \%$ pour les Américains (MOTS, 2019).

5 Les causes d'un tel essor sont connues. D'abord, à condition de réserver via une agence de voyage accréditée par le gouvernement chinois et de visiter en groupe le combiné Singapour-Malaisie-Thaillande, les touristes chinois peuvent depuis 2008 réaliser un séjour dans le pays sans visa (Zhang et Lai, 2009). Ensuite, depuis le $1^{\text {er }}$ janvier 2014, les touristes chinois individuels peuvent demander un visa (payant) directement à leur entrée dans le pays, au passage en douane, sans procédure préalable avant leur départ en Chine (CCA, 2014). Cela représente un avantage incontestable pour la Thaillande, par 
rapport aux autres destinations de la région, pour attirer les touristes chinois : la durée légale du visa est de 60 jours par entrée (des entrées multiples pendant une période de 6 mois sont possibles), alors qu'un séjour à Hong Kong ne peut pas dépasser 7 jours et que de longues procédures administratives sont nécessaires pour visiter le Japon, la Corée du Sud et même Taïwan ; qui plus est, pour des voyages individuels (Deng, 2007). On notera également que depuis quelques années déjà, des vols directs relient la République populaire de Chine à la Thaillande, depuis les grandes villes chinoises mais aussi les villes de taille moyenne.

6 Cette densification des flux a depuis quelques années généré une diversification des pratiquants, en particulier les touristes chinois qui voyagent de façon individuelle. Avant les années 2000, les principaux tour-opérateurs chinois (d'État) proposaient uniquement des circuits touristiques "tout compris" (all inclusive), qui ne laissaient aucune liberté aux touristes en groupes (Li, 2019). Cependant, avec l'émergence des réseaux sociaux et la pratique des achats en ligne, depuis la fin des années 2000, le tourisme chinois en Thaillande est confronté à un nouvel enjeu, celui de l'achat des produits touristiques en ligne. Les principaux tour-opérateurs chinois l'ont bien compris et fournissent maintenant des services en ligne (CAAC, 2018). Parallèlement, de nombreuses jeunes entreprises se sont rapidement positionnées sur ce nouveau segment de marché, jusqu'à devenir des acteurs incontournables. Selon le rapport de l'industrie du tourisme chinois en ligne, réalisé par Yiguan (2016), 5,3\% des produits touristiques ont été réservés en ligne en 2011, contre $15.8 \%$ en 2016. Ctrip fait partie de ces nouvelles entreprises. Fondée en 1999 à Shanghai, c'est aujourd'hui le plus grand fournisseur de services touristiques de Chine. Au début de son activité, l'entreprise permettait la réservation d'hôtels, de billets de transports et de circuits organisés. Aujourd'hui, son modèle combine la vente de services touristiques en ligne pour les particuliers et un réseau de bureaux locaux (en Chine et à l'étranger), où sont proposés des services pour les particuliers, voire pour des agences réceptives.

7 Mais c'est surtout le développement de l'Internet mobile et des réseaux sociaux qui permet la forte émergence actuelle du tourisme chinois individuel. Les touristes chinois, comme les autres touristes du monde, disposent désormais d'outils pour préparer, puis promouvoir leur voyage, sur place et à leur retour (Vincent, 2014). Se prendre en photo est pour les Chinois une pratique touristique qui symbolise la réussite sociale (Taunay et Vacher, 2018). Dès lors, les partages d'expériences en ligne, avec photos et textes (blogue) à l'appui, ou grâce à de courtes vidéos (vlogue), sont devenus des outils touristiques populaires, encouragés par les applications des réseaux sociaux en Chine. L'impact de cette promotion des voyages est particulièrement fort chez les "nouveaux touristes chinois», notamment les jeunes touristes individuels. Sur l'application Wechat (lancée en 2012), ils partagent tous les détails de leurs voyages via leur Pengyou Quan (cercle d'amis; 朋友圈) et deviennent, d'une certaine manière, des influenceurs eux-mêmes. Mais, surtout, depuis 2017, Wechat permet de réserver ses vols, hébergements et produits touristiques directement depuis l'application. De là à dire que cette application, la plus populaire en Chine (près d'un milliard d'utilisateurs), se transforme en site de réservation en ligne, il n'y a qu'un pas. 


\subsection{Des professionnels chinois qui se lancent sur le marché des photos de mariage}

8 On assiste donc à une augmentation régulière des flux de touristes chinois en Thaillande, ce qui peut générer, comme ailleurs dans le monde, des adaptations (Cholat et al., 2019) de la part des acteurs locaux du système touristique, jusqu'alors davantage tournés vers les foyers européens (Hou et al., 2013). Ce sont ces ajustements au marché chinois qui nous intéressent ici; à Phuket en particulier, l'une des principales îles touristiques au sud du royaume. Si nous avons choisi cette île en particulier, c'est qu'elle représente l'une des principales destinations du tourisme chinois dans le pays, comme le montre une analyse réalisée grâce à la méthode développée par Philippe Violier (2011) à partir de tour-opérateurs chinois (Li, 2019). Ceux-ci ne proposent pas toujours un itinéraire précis en ligne, seulement une liste de villes en Thaïlande. L'île de Phuket constitue, la plupart du temps, une seule entité, où les touristes chinois en groupe passent plusieurs jours exclusivement, en différents lieux de l'île.

Une fois sur place, on constate également une forte fréquentation de touristes individuels. Plus particulièrement, lors de deux séjours sur l'île de Phuket, nous avons assisté à une pratique qui nous a interpellé, proposée uniquement aux touristes chinois individuels. Il s'agit de photos de mariage, avant que le mariage n'ait lieu en Chine. Sur place, les clichés n'étaient pas pris par des amis ou des touristes de passage mais par des professionnels, et les touristes chinois portaient quant à eux des costumes (de mariage) loués pour l'occasion. Sur la plage de Kamala en particulier (voir photos 1 et 2), nous avons été plusieurs fois confronté à ce type de pratique, intitulée sur les sites internet des entreprises concernées «Pre-Wedding Photo Shoot». Les couples costumés sont assistés d'une équipe rémunérée pour assurer les séances photos: un photographe, un assistant, une styliste et un chauffeur. 
Photos 1 et 2 . Un cliché sur la plage de Kamala (pendant et après la prise de vue)

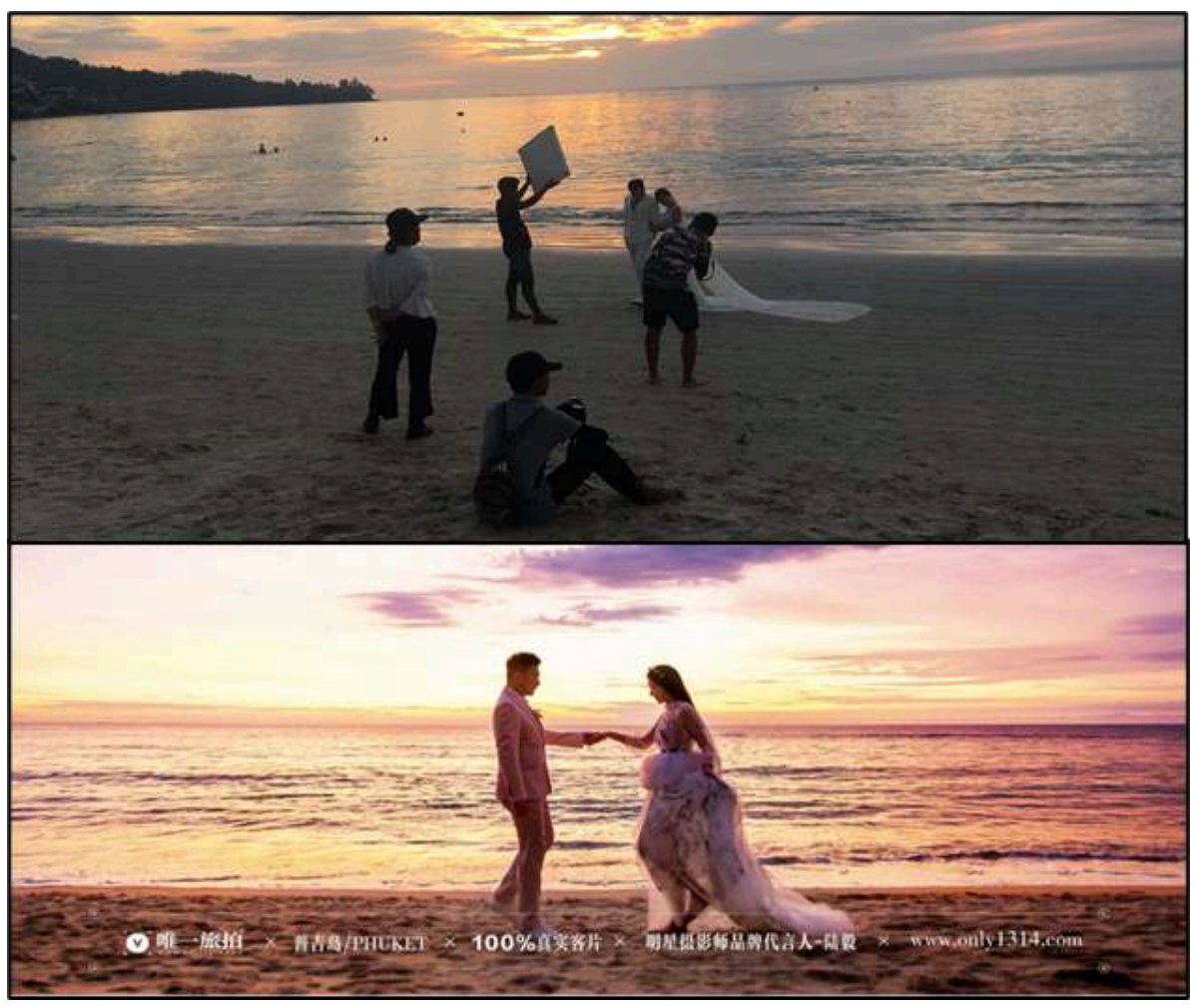

(c) Meng Li, février 2018 (en haut) / site internet d'Onlyphoto (en bas)

10 Au-delà de ce cas particulier, sur différentes plages à Phuket, nous avons à chaque fois observé les regards incrédules des autres touristes présents. Les visiteurs internationaux, occidentaux en particulier, ne cessaient de prendre des photos de cette situation, interrompant leurs activités pour observer ce phénomène. Pourquoi une telle incompréhension devant cette pratique chinoise à Phuket ? Cette manière de faire n'est pourtant pas nouvelle; elle apparaît à Taïwan dès les années 1980, alors dans des ateliers dédiés (Li, 2004). À peu près à la même époque, alors qu'ils constituaient le principal contingent de touristes asiatiques dans le monde, les Japonais usaient eux aussi de cette pratique et l'ont transposée lors de leurs voyages internationaux, sur l'île d'Hawaï notamment (Ricard, 2003). Le phénomène prend également son essor, plus tard, en Chine, comme l'explique Mme Guo, directrice de clientèle au sein de l'entreprise Weiyi : «Les Chinois ne font pas comme les Occidentaux, Ils prennent des photos de mariage avant la cérémonie, pas pendant celle-ci $\|^{1}$. Un documentaire consacré à cette pratique ("China love", sorti en 2018) ${ }^{2}$ montre ainsi de jeunes couples chinois qui se font prendre en photo dans des ateliers à Shanghai, alors même qu'ils ne sont pas encore mariés. Ces ateliers ont fabriqué une série de scènes occidentales, qui sont autant de décors, comme un château, un palais royal, la mer, ou un jardin à la française. Dans le documentaire, on entend que certains clients ont plus de budget que d'autres et choisissent de se rendre soit en Asie du Sud-Est, soit en Europe afin d'y prendre les photos. D'ailleurs, revenant à Kamala, une jeune femme nous a expliqué (début 2018) que "c'est un rêve à réaliser [...] afin de vivre comme dans une vie féérique ». 


\subsection{Méthodologie de recherche : à la rencontre des opérateurs chinois à Phuket}

Plutôt que de penser l'originalité de cette pratique, nos observations nous ont conduit à nous poser les questions suivantes : qui sont les acteurs actifs au sein de cette pratique touristique? Pourquoi et comment s'organisent-ils pour répondre à cette demande touristique individuelle? Pour donner un cadre précis à ces interrogations, nous avons choisi de nous pencher plus particulièrement sur la région de Kamala, à l'ouest de l'île de Phuket. Au cours de deux terrains successifs (en 2018 et en 2019, lors d'un travail de thèse), nous avons en effet constaté que cette zone est celle qui concentre le plus d'ateliers de photos de mariage sur l'île de Phuket: une trentaine, pour les touristes individuels chinois seulement. Nous avons dès lors travaillé, à partir d'observations participantes et d'entretiens semi-directifs, à éclairer les profils de ces professionnels venus spécialement de Chine, leurs manières de travailler sur place et de s'adapter aux pratiques touristiques chinoises, ainsi que les formes spatiales qu'ils ont mises en place à Kamala.

Ce travail a comporté des difficultés. Bien que je sois chinoise, je n'ai pas pu interroger tous les responsables de ces ateliers, rencontrant même de nombreuses réticences de la part de la majorité des photographes. Comme nous le verrons plus bas, la plupart d'entre eux sont en situation de travail illégal et se méfient donc des personnes qu'ils ne connaissent pas (ils ont peur de la police). J'ai dès lors dû passer du temps sur le terrain et construire des relations avec ceux qui ont accepté de discuter avec moi. Les extraits d'entretiens présentés ici sont donc le fruit d'un long effort. Les acteurs chinois n'ont pas souhaité être enregistrés et j'ai dû retranscrire leurs paroles de mémoire, après l'entretien. Afin de contextualiser mais aussi de croiser leurs explications, j'ai mené des entretiens complémentaires avec des acteurs d'autres nationalités travaillant dans ou pour ces entreprises chinoises (cf. tableau 1). L'ensemble représente un total de 670 minutes d'entretien. Enfin, pour compléter cet échantillon, je m'appuie également sur d'autres entretiens, menés à Shanghai, au siège de compagnies chinoises ayant ouvert une agence à Phuket.

Tableau 1. Acteurs interrogés dans le champ des photos de mariage à Phuket

\begin{tabular}{|c|c|c|c|c|c|}
\hline $\begin{array}{l}\text { Nom } \\
\text { (modifié) }\end{array}$ & Nationalité & Date & $\begin{array}{l}\text { Durée de } \\
\text { l'entretien }\end{array}$ & Sexe & Profession \\
\hline Momo & Chinoise & $\begin{array}{l}9 \text { janvier } \\
2018 \\
16 \text { janvier } \\
2018 \\
9 \text { février } \\
2018 \\
3 \text { février } \\
2019 \\
19 \text { février } \\
2019\end{array}$ & $300 \mathrm{mn}$ & Femme & $\begin{array}{l}\text { Directrice d'un atelier de } \\
\text { cérémonies de mariage }\end{array}$ \\
\hline
\end{tabular}




\begin{tabular}{|c|c|c|c|c|c|}
\hline $\mathrm{Lu}$ & Chinoise & $\begin{array}{l}18 \text { janvier } \\
2018 \\
9 \text { février } \\
2018\end{array}$ & $120 \mathrm{mn}$ & Homme & Photographe \\
\hline Yanan & Chinoise & $\begin{array}{l}18 \text { janvier } \\
2018\end{array}$ & $30 \mathrm{mn}$ & Femme & Styliste \\
\hline John & Canadienne & $\begin{array}{l}18 \text { janvier } \\
2018\end{array}$ & $30 \mathrm{mn}$ & Homme & $\begin{array}{l}\text { Animateur de cérémonies de } \\
\text { mariage }\end{array}$ \\
\hline Pann & Thaillandaise & $\begin{array}{l}18 \text { janvier } \\
2018\end{array}$ & $20 \mathrm{mn}$ & Femme & $\begin{array}{l}\text { Manageur de salle des fêtes } \\
\text { (une fausse église pour les } \\
\text { cérémonies de mariage) }\end{array}$ \\
\hline Wang & Chinoise & $\begin{array}{l}18 \text { janvier } \\
2018\end{array}$ & $20 \mathrm{mn}$ & Homme & Photographe \\
\hline Zhang & Chinoise & $\begin{array}{l}18 \text { janvier } \\
2018\end{array}$ & $20 \mathrm{mn}$ & Homme & Photographe \\
\hline Tim & Canadienne & $\begin{array}{l}19 \text { février } \\
2019\end{array}$ & $30 \mathrm{mn}$ & Homme & $\begin{array}{l}\text { Animateur de cérémonies de } \\
\text { mariage }\end{array}$ \\
\hline Nic & Laotienne & $\begin{array}{l}19 \text { février } \\
2019\end{array}$ & $20 \mathrm{mn}$ & Homme & Factotum de cérémonie \\
\hline Liao & Laotienne & $\begin{array}{l}31 \text { janvier } \\
2018\end{array}$ & $20 \mathrm{mn}$ & Homme & Factotum de cérémonie \\
\hline Guo & Chinoise & $\begin{array}{l}7 \text { décembre } \\
2018\end{array}$ & $60 \mathrm{mn}$ & Femme & $\begin{array}{l}\text { Directrice de clientèle chez } \\
\text { Weiyi }\end{array}$ \\
\hline
\end{tabular}

\section{Comment travaillent les professionnels chinois, pour les photos de mariage, à Phuket?}

\subsection{Qui sont ces professionnels des photos de mariage ? Comment en sont-ils venus à travailler à Phuket?}

On trouve plusieurs types d'entreprises et de professionnels travaillant dans le champ des photos de mariage à Phuket. En premier lieu, ce sont des entreprises chinoises qui ont ouvert une agence en Thaïlande. Comme l'avance Mme Guo, des entreprises ont inventé l'idée de "prendre les photos de mariage en voyageant». Celle dans laquelle elle travaille propose d'ailleurs ses services dans plusieurs pays étrangers, en Thaïlande notamment. Lors des congés chinois, le nouvel an en particulier (entre fin janvier et début février chaque année), plusieurs employés sont mobilisés et envoyés travailler à l'étranger. Dans le cas de Phuket, l'entreprise a sélectionné différents lieux de tournage et plusieurs circuits sont proposés aux clients qui ont les moyens de se payer ces formules. Les tarifs et logiques de ces séjours nous permettent de mieux comprendre 
les pratiques et l'organisation spatiale déployées pour les touristes chinois (quasiment tous des individuels). Trois forfaits existent : le premier à 9999 yuans (1 283 euros) avec trois sites de photos au choix; le second à 12999 yuans (1 669 euros) avec six sites au choix ; le dernier à 16999 yuans (2 182 euros) avec sept sites possibles. Chaque forfait se décline sur une à trois journées, selon le temps disponible des touristes. Les sites sont tous concentrés dans la zone de Kamala. Le forfait A (cf. encadré 1) est, selon nos entretiens sur place, le plus populaire. Il assemble des prises de photos devant la mer, dans une rue urbaine thaïlandaise et dans une villa de luxe. L'ensemble forme une image variée : la mer représente le plaisir des «vacances occidentales à la plage », la rue thaïlandaise offre l'ambiance « exotique » de l'Asie du Sud-Est, tandis que la villa de luxe montre un style de vie "privilégié» (pour reprendre les termes utilisés sur le site internet de l'entreprise: m.only1314.com). Le tout forme une mise en scène que les touristes pourront rarement reproduire dans leur vie quotidienne, après le mariage.

Encadré 1. Les forfaits et les sites de tournage de Onlyphoto

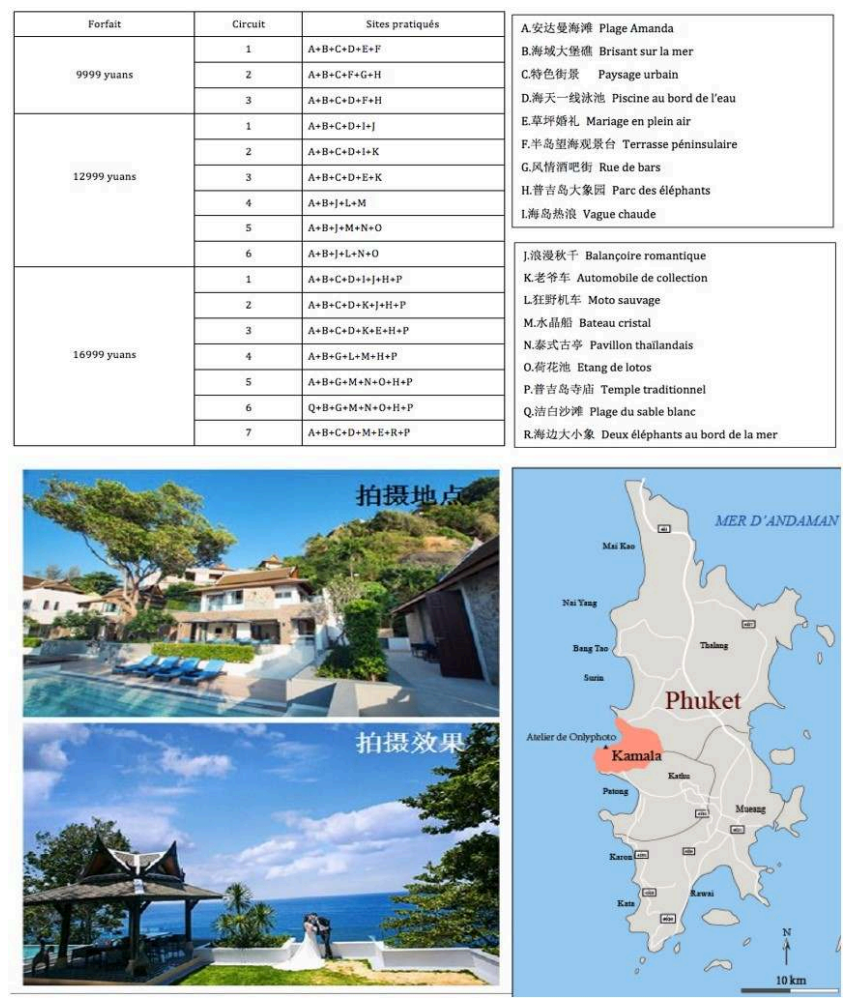

Après leurs journées consacrées à la prise de clichés (photo 4, en bas), les touristes individuels retournent se reposer dans une villa (photo 3, en haut) située à Kamala (carte 1).

Réalisation personnelle (2019)

Cette organisation est facilitée par la situation de la Thaïlande par rapport à la Chine en général, et de Phuket en particulier. L'absence de visa (obtenu directement à l'arrivée sans formalités) fait qu'il est aisé de s'y rendre. Selon le rapport sur le voyage individuel, publié par Mafengwo (2016), Phuket représente, par rapport aux autres destinations insulaires en Chine - et pour le même budget -, un service de meilleure qualité, une ambiance plus internationale, un accueil souriant. Tout ceci fait que les jeunes couples préfèrent de plus en plus se rendre à Phuket plutôt que sur l'île de Hainan pour prendre leurs photos de mariage. D'ailleurs, on note que ces touristes ont eu des expériences de voyage à l'étranger plus nombreuses que les groupes de touristes 
chinois, qui se déplacent avec un guide, par l'intermédiaire de tour-opérateurs. Ces touristes individuels ont étudié leur projet de voyage avant le départ, ou ils ont sollicité l'aide d'un guide privé. Ils viennent sur l'île de Phuket avec des objectifs clairs et ce sont souvent eux que l'on retrouve sur la plage, par exemple pour découvrir la pratique $\mathrm{du}$ repos (Li, 2019).

Le deuxième type d'acteurs chinois rencontré à Kamala est majoritairement constitué de petits entrepreneurs chinois venus de Hainan. Ils sont certes arrivés après les grands groupes décrits ci-dessus, mais ils représentent aujourd'hui la majorité de l'offre sur place ( $c f$. tableau 2). Ainsi, la plupart des agences officiant localement dans le secteur des photos de mariage sont de petite dimension. Un photographe interrogé en 2018, M. Lu, en atteste : «il y a environ 30 ateliers de photos de mariage sur l'île de Phuket. Ils sont tous tenus par des Chinois et sont de différentes tailles: les grandes entreprises chinoises, les ateliers moyens et les photographes individuels. Ils sont concentrés dans la région de Kamala $»^{3}$. Tous ont profité de l'accessibilité de plus en plus aisée de la Thaïlande en général, et de Phuket en particulier, pour venir s'installer sur place et y ouvrir une agence.

Tableau 2. Les différents types d'entreprises de photos de mariage chinoises à Phuket

\begin{tabular}{|l|l|}
\hline Type & Nombre \\
\hline Grandes chaînes de photos de mariage chinoises & 2 \\
\hline Grands studios & 7 \\
\hline Ateliers indépendants & Plus de 20 \\
\hline Photographes indépendants & Inconnu \\
\hline
\end{tabular}

Réalisation personnelle (2019)

En ce sens, l'expérience et l'histoire de cet homme constituent quasiment un idéal-type de la situation à Phuket. En Chine, M. Lu était photographe dans un studio de photos de mariage à Pékin et à Sanya (où il était envoyé saisonnièrement). Il a débarqué sur l'île de Phuket en 2014, à la demande de sa société, pour une mission qui a duré deux mois : "Quand je suis arrivé en Thailande, j'utilisais un visa touristique qui ne me permettait de rester que pendant un mois. Puis, j'ai renouvelé mon visa le mois suivant. Je pense que les affaires, pour les photos de mariage, permettent de gagner plus d'argent à Phuket qu'en Chine $»^{4}$. Après avoir travaillé à Phuket pendant un certain temps, il a quitté sa société et a commencé à prendre ses propres commandes avec sa petite amie (styliste, en charge du maquillage et de la coiffure dans leur atelier actuel): «Beaucoup de photographes indépendants commencent de cette façon $»^{5}$. Au bout de six mois, ils ont loué une villa et créé leur atelier de photos de mariage. Il est situé dans un quartier résidentiel, sur le côté est de la route principale menant à la plage de Kamala.

Cette localisation n'est pas anodine: l'atelier n'est pas situé sur les plages les plus fréquentées de l'ouest de l'île de Phuket (comme Patong, Karon ou Kata). Il se trouve, au contraire, dans le quartier localisé au sud-ouest de la plage de Kamala, là où sont réunis les resorts de luxe, sorte d'écrin plus intime et plus calme que les autres quartiers à proximité. Le lieu originel de cette pratique touristique originale et spécifique aux 
touristes chinois individuels se trouve d'ailleurs être la plage de Kamala, comme nous l'ont précisé plusieurs membres des différents ateliers lors de nos séjours: "Des amis m'ont présenté cet endroit. Tout le monde prend une photo de mariage à Kamala. Les grandes entreprises comme Platinum et Weiyi ont loué des villas sur les falaises à l'ouest de la région de Kamala. Il existe probablement plus de 30 petites entreprises et studios comme moi, tous situés dans l'est de Kamala $»^{6}$. Un agent immobilier (Jas) évoque d'autres raisons : «Parce que le terrain est élevé, les villas sont sur les falaises et le paysage marin est particulièrement beau. Près de l'aéroport [situé à 20 kilomètres au nord de Kamala], il est possible d'obtenir un emplacement plus privé [il y a moins de touristes sur la plage] que dans la région de Patong. Il y a beaucoup de villas de luxe et de nombreuses villas accueillent des célébrations de mariage haut de gamme. Donc, les entreprises chinoises sont ici $»^{7}$.

La villa de M. Lu est exemplaire du fonctionnement des autres entreprises de même taille. Elle comporte deux niveaux, chacun d'une superficie d'environ $100 \mathrm{~m} 2$. Le rezde-chaussée constitue la salle de réception ainsi que l'espace où sont rangés les costumes de mariage. Le premier étage sert aux chambres des clients. Tout le design intérieur et la décoration ont été réalisés par leurs soins : «Lorsque nous avons commencé à préparer ce studio, nous avions seulement le matériel de photographie. Tous les costumes de mariage et les décorations de l'atelier sont importés de Chine $»^{8}$. En revanche, qu'il s'agisse d'une grande entreprise de photographie de mariage ou d'un atelier individuel, le schéma d'une journée est relativement identique d'une boutique à l'autre, pour satisfaire les désirs des clients et les faire se sentir, autant que possible, comme en Chine: "Les clients habitent dans notre villa, mais nous ne proposons pas trois repas quotidiens. Certains ateliers offrent des repas chinois à leurs clients. Nous, nous faisons plus attention aux photos ${ }^{9}$. D'ailleurs, la stratégie qui unit les principaux ateliers, à la différence des indépendants, est la suivante : «Les grandes entreprises ont une source fixe de clients. Leurs bureaux en Chine leur envoient des clients régulièrement. Les ateliers individuels doivent eux trouver seuls les clients grâce aux réseaux sociaux. La concurrence est féroce ${ }^{10}$. Aucun des entrepreneurs chinois de Kamala n'a d'ailleurs accepté de nous montrer l'emplacement des autres ateliers. La plupart d'entre eux, y compris les grandes entreprises, ne disposent pas de panneaux de signalisation: en effet, leurs propriétaires n'ont pas officiellement créé d'entreprise avec des résidents thailandais, ils sont donc en situation illégale.

\subsection{Pourquoi et comment exportent-ils leur expérience à Phuket ?}

Comme d'autres photographes chinois rencontrés à Kamala, M. Lu explique que «la chose la plus importante à faire dans ce travail est de se protéger contre la police. La police thailandaise est comme un loup, ils sont comme des loups affamés $»^{11}$. C'est que, comme nombre d'autres entrepreneurs chinois, il est arrivé en Thaillande avec un visa touristique et a commencé à travailler avec un visa étudiant d'un an (obtenu via une école de langues, tenue par une Chinoise qui a immigré à Phuket, Mme Zhang). Mais cette solution est périlleuse : après un long séjour, les autorités douanières ont constaté que son passeport ne présente que des allers-retours entre la Thaillande et la Chine. Comme il nous l'a confié, s'il ne soudoyait pas de temps en temps la police, elle ne le laisserait pas passer à chaque entrée à l'aéroport de Phuket. Les douanes savent cependant que les propriétaires de ces entreprises travaillent illégalement en Thaïlande et qu'ils ne sont normalement pas autorisés à passer la douane avec leur visa étudiant : «Les douanes savent que les personnes comme nous font la navette entre Phuket et la 
Chine, que ce sont des travailleurs illégaux, les photographes et les guides touristiques. Ils attendent que nous les corrompions avec de l'argent. Nos équipements photographiques et nos costumes étant chers, ils se servent de cet argument lorsqu'ils nous conduisent à la salle d'examen à l'aéroport. Lorsque la police m'a emmené dans la salle d'examen pour étudier mon équipement, j'avais déjà préparé l'argent, dans mes manches, 300 à 500 yuans [entre 40 et 60 euros]. En entrant dans la salle d'inspection, je l'ai secrètement remis à la police des douanes $»^{12}$. Nous expliquant cela, il nous montre comment la police collecte l'argent : "Ils vont rapidement mettre l'argent dans leur livret [leur carnet d'observations et de contrôle des équipements], cette action est très habile ». Et cette situation est récurrente : "Nous devons le faire à chaque fois, tout le monde fait comme ça " ${ }^{13}$.

Les contrôles ne s'arrêtent cependant pas à l'arrivée à l'aéroport, les douanes et les forces de police travaillent ensemble : «Elles contrôlent souvent les sociétés de photographie et les studios. Elles ont contrôlé l'entreprise Platinum il y a quelques jours. C'est pourquoi celle-ci ne peut pas travailler pendant quelques jours $»^{14}$. M. Lu a lui aussi été expulsé vers la Chine en raison de son activité illégale de photographie, fin 2018. Il est pourtant revenu fin mars 2019, comme nous l'avait prédit Momo, la directrice d'un atelier de cérémonies de mariage, quelques jours plus tôt : «Après son arrestation, Lu a été tout de suite inscrit sur la liste noire locale et a été envoyé au poste de police de Bangkok pour un rapatriement direct en Chine. Le seul moyen pour qu'il revienne est de changer de nom, puis de changer son passeport pour revenir en Thailande $»^{15}$. M. Lu a justement utilisé cette méthode et, fin mars 2019, il était de retour dans son studio à Phuket. Pendant son absence, le travail de son entreprise n'a pas cessé, sa petite amie se chargeait de contacter les clients chinois et le travail de photographie était réalisé par un de leurs amis. En mai 2019, toutefois, leur atelier de photos de mariage a déménagé à Bali, en Indonésie - signe qu'il n'est pas aisé de s'installer durablement sur ce marché.

\section{Des hiérarchies entre les employés touristiques de la photo de mariage}

\subsection{Des employés occidentaux au service des entrepreneurs chinois}

21 Toutefois, si les migrants chinois arrivent parfois à perdurer dans cette activité de photographie de mariage, c'est qu'ils font travailler une partie de la société locale ; des Thaïlandais, mais aussi d'autres migrants des pays voisins. Les acteurs chinois, parfois les derniers arrivés comme nous l'avons vu, se sont ainsi rapidement adaptés à la situation locale. Les entrepreneurs chinois embauchent même parfois des résidents occidentaux de l'île. Tous profitent des opportunités d'emploi générées par l'arrivée des touristes chinois. Les migrants d'Asie du Sud-Est, auparavant souvent serveurs dans les restaurants, employés dans des hôtels, etc., travaillent désormais volontiers au sein de l'industrie des cérémonies de mariage. 
Photos 5 et 6 . La chapelle de mariage de la région de Laguna

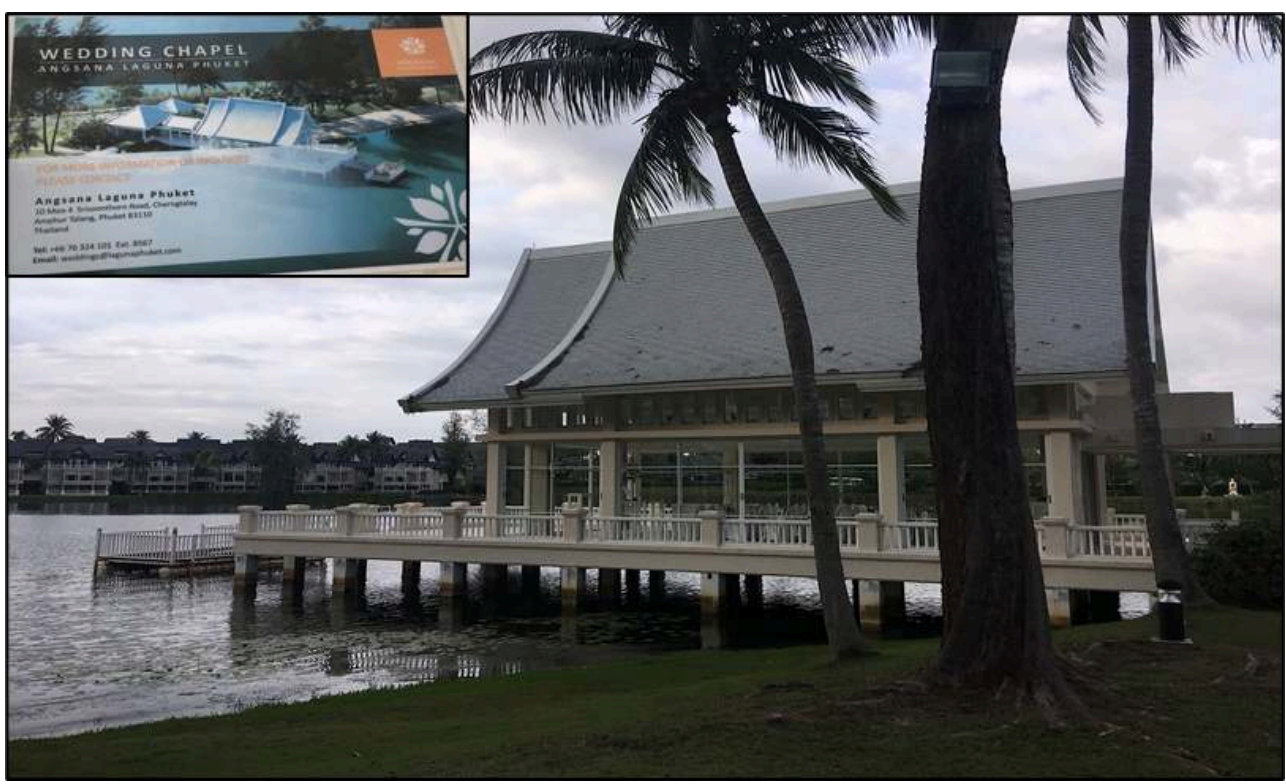

(c) Meng Li, janvier 2018

Nous avons pu observer une de ces cérémonies, au sein de la chapelle « Angsana Laguna Phuket », en janvier 2018. Celle-ci se trouve dans un jardin au bord d'un lac, au milieu des nombreuses villas privées situées dans la région de Laguna, au nord de l'île de Phuket (photos 5 et 6): "Cette chapelle a été construite par une entreprise japonaise, spécialement pour les cérémonies de mariage. Son architecte est japonais. Les affaires n'étaient pas très bonnes jusqu'à ce que le groupe Angsana l'achète $»^{16}$. Pour donner un caractère international à la cérémonie, les acteurs chinois font appel à des Occidentaux. C'est le cas de John, Canadien et résidant sur l'île de Phuket depuis 25 ans : "Je suis venu ici dans les années 1990. Au début, j'ai travaillé comme guide privé pour les Canadiens et les touristes anglophones. [...] Maintenant, j'anime les cérémonies de mariage dans cette chapelle. C'est ainsi que je complète mes revenus pendant ma retraite ${ }^{17}$. Selon lui, le déroulement de tous les offices destinés aux touristes chinois est le suivant: "C'est une cérémonie américaine. La mariée entre avec son père, le marié l'attend devant moi dans la chapelle. Ils font le serment de mariage selon mes consignes. À la fin, ils signent un certificat de mariage en anglais. Il n'a pas de légalité officielle en tant que mariage civil. C'est juste une étape formelle. La routine de la cérémonie est planifiée par le responsable de chapelle [un Thaillandais]. Mais l'ordre des étapes peut changer si les clients sont pressés. En tout cas, moi, je prononce toujours les mêmes paroles $^{18} \|^{19}$. Comme d'autres acteurs du système de photos de mariage à Phuket, il a lui aussi remarqué le changement de clientèle qui s'opère depuis quelques années: "Auparavant, les mariés venaient souvent de Singapour, du Japon ou d'Australie. La chapelle travaille avec Momo depuis trois ans, le nombre de clients chinois augmente énormément. Elle organise au moins deux à trois cérémonies pour les Chinois par mois. Comparés aux autres mariés, les Chinois font très attention à l'enregistrement de la cérémonie. C'est-à-dire qu'ils profitent moins du moment de la cérémonie avec leurs invités, ils préfèrent filmer les scènes de la cérémonie. Certains couples chinois n'invitent même pas leur famille et leurs amis. Ils me demandent souvent de prendre beaucoup de photos de la scène du serment ou de la signature du certificat. Je le fais avec plaisir. Je comprends bien qu'ils me considèrent comme un symbole pour leur cérémonie de mariage occidentale $»^{20}$. 
Lors d'une autre cérémonie de mariage, cette fois en plein air, sur la falaise au sud de Patong, Momo nous apprend que : «le propriétaire du terrain de mariage est Chinois. Il a ouvert cet endroit spécialement pour les cérémonies de mariage des Chinois. Cet emplacement est simple mais la vue est magnifique. Il est moins cher que si cela se passait à l'hôtel. On travaille ensemble depuis trois ans. Le patron m'a fait un tarif préférentiel $»^{21}$. Comme on le voit sur le cliché suivant (photo 7), les installations du site sont simples: un abri à droite (où attendent deux employés birmans), les mariés autour de l'animateur (canadien) de la cérémonie, ainsi que les amis (chinois) du couple, dont une prend une photo (au premier plan, au centre). Ici, tous les employés cités travaillent pour des opérateurs chinois. Ces derniers supervisent la mise en scène avec les employés birmans (qui réalisent effectivement le travail) selon les différentes exigences du client. Ce lieu se développe petit à petit : il existe des petites entreprises de location de quads (motos à quatre roues), situées à proximité, à destination des touristes chinois en groupe ${ }^{22}$.

Photo 7. Cérémonie de mariage à proximité de Patong, février 2019

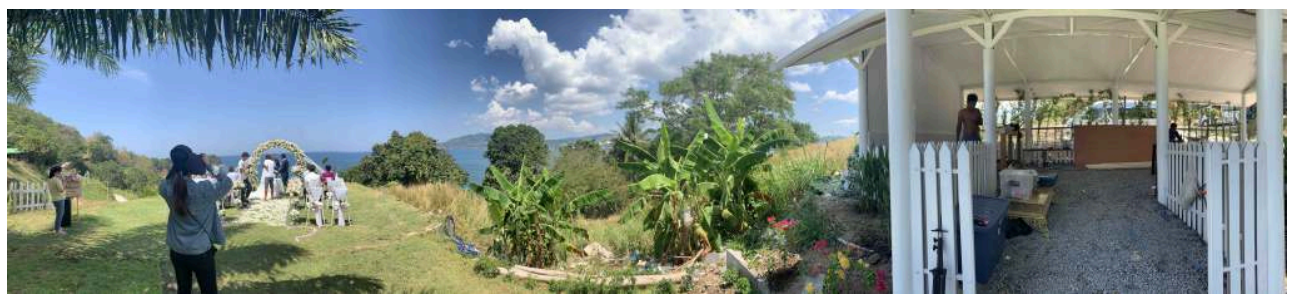

(c) Meng Li, février 2019

À l'issue de la célébration, nous avons interrogé Tim, l'animateur de cette cérémonie de mariage. Il a 42 ans, vient de l'est du Canada et est ici avec sa famille depuis plus de cinq ans. Il était ingénieur auparavant. Maintenant, il travaille en tant que professeur d'anglais et officie également comme animateur pour des cérémonies de mariage comme celle-ci. Un opérateur chinois l'a contacté sur Facebook et ils ont commencé à travailler ensemble. Depuis, il a également travaillé avec d'autres sociétés chinoises. En effet, Tim est très demandé : « son âge et son apparence conviennent très bien à l'image que se font les Chinois d'un prêtre »" De son côté, Tim raconte: "Ma femme est photographe. Elle est venue une fois travailler à Phuket. Nous sentions tous les deux que la vie à Phuket était très agréable, alors nous avons décidé d'y rester. Nos deux enfants sont nés à Phuket. Je suis devenu professeur d'anglais. Par la suite, j'ai travaillé comme animateur de cérémonies de mariage, organisées par des grands hôtels. Les clients étaient souvent Australiens ou Indiens. J'anime aussi la réunion annuelle de la société des photographes. Après que Momo m'a contacté sur Facebook, j'ai commencé à travailler avec les Chinois »²4.

\subsection{Des immigrés d'Asie du Sud-Est en renfort}

25 À l'issue de cette cérémonie, Tim s'est vu remettre la somme de 5000 baths (environ 150 euros), un montant qui correspond au salaire mensuel des employés birmans (photo 8). Cela illustre la hiérarchie claire existant entre les employés non chinois qui travaillent avec le tourisme chinois. En ce sens, la main-d'œuvre bon marché non thaïlandaise du sud-est de l'Asie constitue l'un des moyens utilisés par certains employeurs chinois pour réduire les coûts et augmenter les profits. Et ceci dépasse le seul cadre des entrepreneurs chinois prenant des photos de pré-mariage à Phuket. 
Photo 8. Un employé birman fait le ménage après la cérémonie de mariage

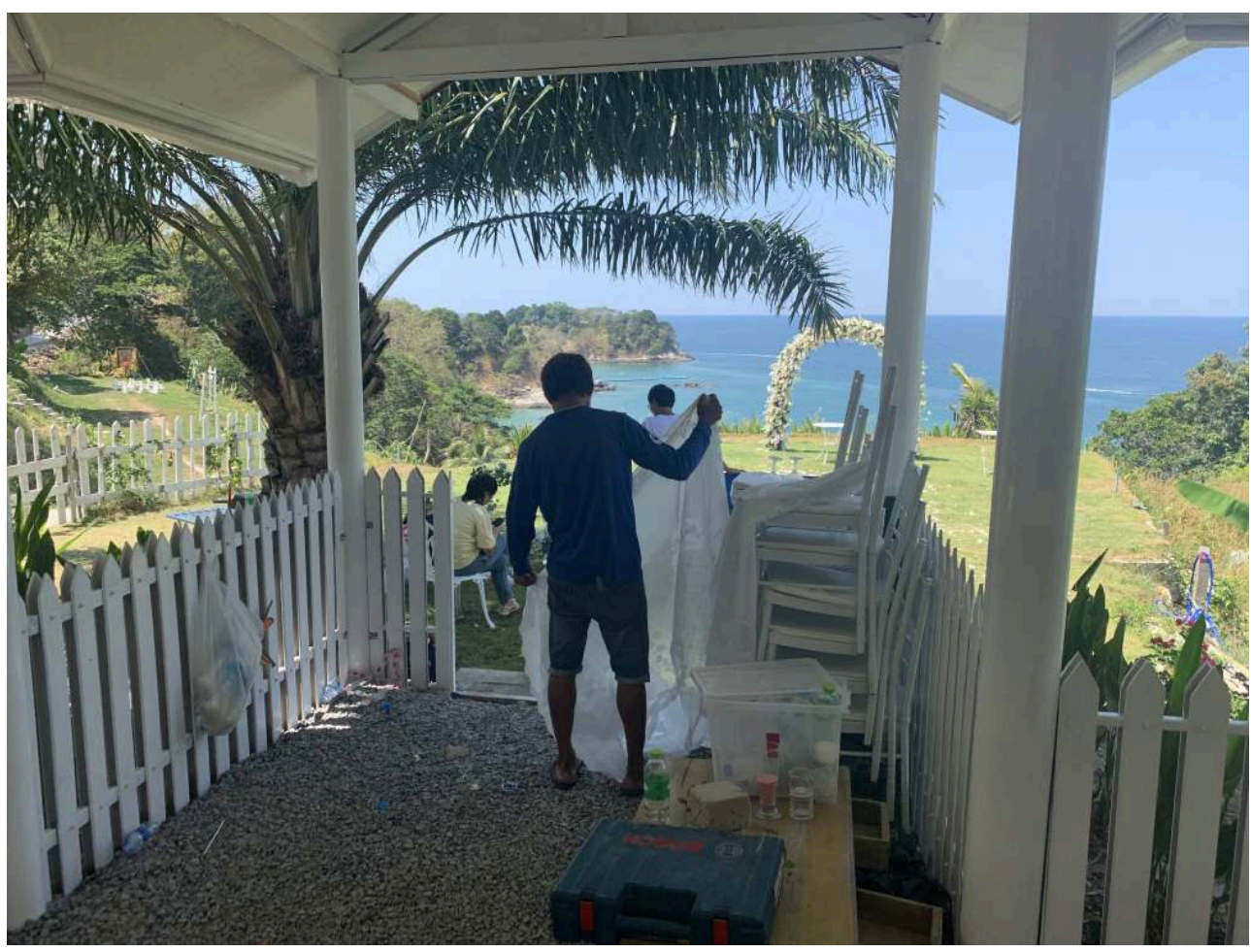

(C) Meng Li, février 2019

En effet, selon les entretiens que nous avons menés avec d'autres petits entrepreneurs chinois dans d'autres secteurs, tous préfèrent recruter des employés birmans ou laotiens plutôt que des Thaïlandais. M. Zhang, propriétaire (chinois) d'un restaurant chinois à Patong, explique à ce sujet que : «D'abord, je voulais avoir des serveurs qui parlent chinois. Parce que la plupart des clients chez nous sont chinois. Mais un serveur chinois avec un permis de travail ou un serveur thailandais qui parle chinois coûtent très cher. Je peux engager deux employés laotiens pour le même salaire. Ils peuvent apprendre le chinois de base pendant le travail $\aleph^{25}$. Dans un secteur d'activité proche, M. Pan, propriétaire d'une maison d'hôte située à Patong, s'accorde avec cette façon de faire : "J'ai deux employés laotiens. Ils sont en couple, l'un fait le jardinage, l'autre fait le ménage des chambres. Ils ne travaillent pas mal et leurs salaires sont moins élevés que ceux des Thailandais. Beaucoup de patrons chinois font la même chose $»^{26}$.

Les travailleurs migrants comprennent bien cette logique commerciale. Ils apprennent le mandarin (de base) pour s'adapter rapidement aux touristes chinois. Dès qu'ils en ont la possibilité, ils choisissent même de changer de poste pour obtenir un meilleur salaire. Zaw, par exemple, est un serveur (laotien) travaillant dans un restaurant de fruits de mer chinois, situé dans la rue de Rathuhit à Patong. Il nous raconte : «J'ai travaillé six mois au marché de fruits de mer Bazaan. Ils ont énormément de touristes chinois tous les jours. J'ai appris les noms des fruits de mer en chinois. En plus, je peux dire couramment les chiffres en chinois pour négocier le prix. Cela me permet d'avoir ce poste aujourd'hui. Je gagne plus de 2000 baths [environ 60 euros] par mois ${ }^{27}$. Mais la plupart des travailleurs migrants d'Asie du Sud-Est ne parlent ni anglais ni thaïlandais. Ils occupent les postes les plus difficiles et il n'est pas aisé de les interroger. Certains d'entre eux n'ont même pas de permis de travail. Ils ont été indifférents à nos questions. Il est donc difficile de 
connaître leur situation autrement que par les enquêtes menées auprès des opérateurs chinois qui les emploient. Ces migrants travaillent et leur emploi n'est pas stable (ils sont la plupart du temps en situation illégale). Ils peuvent également être concurrencés par d'autres professionnels du secteur de la photo de mariage, originaires comme eux des pays voisins de la Thaillande, qui pourraient accepter des salaires encore plus bas.

\section{Conclusion}

À l'issue de cet article, on peut dire que c'est un véritable miroir de la hiérarchie touristique qui se (re)dessine à Phuket. Les touristes chinois font l'objet de toutes les attentions, si bien que nombre de petits entrepreneurs chinois ont déménagé de Hainan à Phuket, pour tirer profit d'opportunités économiques réelles. Même si elles ne sont pas forcément pérennes, et pas toujours complètement légales, elles permettent à ces nouveaux acteurs, parmi les derniers arrivés sur place, de monter des entreprises rentables. Plus encore, les entrepreneurs chinois renversent parfois les anciennes hiérarchies touristiques, allant jusqu'à transformer les anciens maitres des lieux (les professionnels occidentaux) en employés, à leur service. Un tel constat ouvre inévitablement de nouvelles pistes de recherche, bien au-delà des seules photos de mariage. Quelles sont, par exemple, les perceptions des employés locaux implantés depuis plusieurs décennies et qui doivent, parfois, céder leur place aux nouveaux venus? Mais aussi, comment s'organise la bataille économique visant à attirer ces touristes chinois? À l'inverse, existe-t-il des stratégies visant à contrer cette avancée de la présence chinoise à Phuket et, au-delà, en Asie du Sud-Est? Une série de questions, non exhaustive, qui nous montre que nous sommes encore bien loin d'avoir épuisé la compréhension du tourisme international chinois.

\section{BIBLIOGRAPHIE}

Wolfgang Georg ARLT, China's Outbound Tourism, Routledge, 2006 [https://doi.org/ $10.4324 / 9780203968161]$.

CAAC, « Rapport annuel », Administration de l'aviation civile de Chine, 2018.

Florent CHOLAT, Luc GWIAZDZINSKI, Céline TRITZ et John TUPPEN, « Tourisme(s) en mouvement(s) entre adaptation, résilience et changement de paradigme ", dans Florent Cholat, Luc Gwiazdzinski, Céline Tritz et John Tuppen, Tourisme(s) et adaptation(s), Elya, 2019 [https:// halshs.archives-ouvertes.fr/halshs-02064504/document].

Bin DAI, Yiyi JIANG, Liqiong YANG et Yiliang MA, « China's Outbound Tourism. Stages, Policies and Choices ", Tourism Management, $n^{\circ}$ 58, 2017.

Zhou DENG, «中国旅游制度 : 历史、变革与发展 》 [Le système de vacances en Chine : histoire, évolution et développement], Édition de l'Université d'études internationales de Pékin, 2007. 
Jiang DU, 《中国旅行社发展回顾和前瞻 》 [Retrospect and Prospect of the Development of China's Travel Service Industry], Tourism Tribune China, $\mathrm{n}^{\circ}$ 6, 2003.

Jean-Christophe GAY, Un coin de paradis. Vacances et tourisme en Nouvelle-Calédonie, Édition de la Ville de Nouméa, 2017.

GÉOCONFLUENCES, « Acteurs spatiaux, action spatiale », 2020 [http://geoconfluences.ens-lyon.fr/ glossaire/acteurs-spatiaux-action-spatiale].

Zhiqiang HOU, Xu Hong FANG et Cuilan ZHU, «中国大陆游客对泰国旅游感知研究——基于刚络自 媒体的内容分析 » [About the Sense of Tourists from China’s Mainland in Thailand: Based in the Content Analysis of the Web Media], China Academic Journal, n 3, 2013.

Meng LI, Processus d'adaptation et logiques d'acteurs face au développement du tourisme chinois en Thailande. Le cas de l'île de Phuket, thèse en géographie, Université d'Angers, 2019 [https:// tel.archives-ouvertes.fr/tel-03082014].

Yuying LI, 《裝扮新娘一當代台灣婚紗業的興起與發展歷史》[The Bride Maker: The Formation and Development of the Bridal Industry in Taiwan], Feng Chia Journal of Humanities and Social Sciences, $\mathrm{n}^{\circ} 8,2004$.

MAFENGWO, 《全球自由行报告》 [Rapport sur le voyage individuel], Mafengwo, 2016 [http:// www.mafengwo.cn/gonglve/zt-735.html].

MOTS, Report of International Tourists Arrivals to Thailand, Ministry of Tourism \& Sports (Thaillande), 2018 [https://www.mots.go.th/more_news_new.php?cid=527].

Alain RICARD, Hawaii, Karthala, 2003.

Benjamin TAUNAY et Luc VACHER, «Pratiques et organisation spatiale de la plage de Dadonghai à Sanya (île de Hainan, Chine) », Mappemonde, n 123, 2018 [https://doi.org/10.4000/mappemonde. 389].

Akarapong UNTONG, Vicente RAMOS, Mingsarn KAOSA-ARD et Javier REY-MAQUIEIRA, « Tourism Demand Analysis of Chinese Arrivals in Thailand ", Tourism Economics, vol. 21, n 6, 2015 [https://doi.org/ $10.5367 /$ te.2015.0520].

Johan VINCENT, «L'appropriation des nouvelles technologies de la mobilité par le tourisme : nouveaux enjeux créatifs », Mondes du tourisme, n 10, 2014 [https://doi.org/10.4000/tourisme. 380].

Philippe VIOLIER, «Les lieux du monde », EspacesTemps.net, 2011 [https:// www.espacestemps.net/articles/les-lieux-du-monde/]

VISA SERVICE OF THAILAND, China Consult Affairs, 2014 [http://cs.mfa.gov.cn/zggmcg/ljmdd/ yz_645708/tg_647570/rjjl_647580/].

\section{NOTES}

1. Entretien avec Mme Guo, le 8 décembre 2017. Weiyi («唯一» en chinois, "Only Photo » en anglais), est une entreprise chinoise spécialisée dans ce type de photographie, localisée dans un ancien site industriel reconverti en quartier artistique, à Shanghai (http://www.only1314.com/ index).

2. «China Love » est un documentaire sorti en 2018, réalisé par Olivia Martin-McGuire : https:// chinalovefilm.com/ 
3. Premier entretien avec M. Lu, le 18 janvier 2018.

4. Deuxième entretien avec M. Lu, le 9 février 2018.

5. Ibid.

6. Ibid.

7. Entretien avec Jas, le 16 janvier 2019.

8. Deuxième entretien avec M. Lu, le 9 février 2018.

9. Ibid.

10. Ibid.

11. Ibid.

12. Entretien avec M. Wang, le 18 janvier 2018.

13. Ibid.

14. Entretien avec Momo, le 20 février 2019.

15. Ibid.

16. Ibid.

17. Entretien avec John, le 18 janvier 2018.

18. "I take you, to be my husband (wife), to have and to hold from this day forward, for better for worse, for richer or poorer, in sickness, and in health, to love and to cherish, as long as we both shall live. This is my solemn vow and promise. " Il s'agit d'un passage connu de la plupart des touristes chinois, en raison des nombreux films américains projetés en Chine reprenant cette formule.

19. Entretien avec John, le 18 janvier 2018.

20. Ibid.

21. Entretien avec Momo, le 20 février 2019.

22. Sur place, nous avons également appris qu'il existe d'autres endroits aux alentours où les Japonais célèbrent des mariages.

23. Entretien avec Momo, le 20 février 2019.

24. Entretien avec Tim, le 20 février 2019.

25. Entretien avec M. Zhang, le 10 février 2019.

26. Entretien avec M. Pan, le 16 février 2019.

27. Entretien avec Zaw, le 13 février 2019.

\section{RÉSUMÉS}

Faisant le constat de l'augmentation régulière du tourisme chinois en Thaillande, cet article se penche sur l'émergence d'une nouvelle catégorie d'acteurs touristiques, sur l'île de Phuket en particulier. Une série d'entrepreneurs vient s'y installer pour y ouvrir des ateliers de photographies de "pré-mariage", une pratique à mi-chemin entre le voyage de noces et un séjour à thème. Ce texte vise à comprendre ce phénomène, tout en ouvrant la discussion sur la constitution d'un système économique qui l'organise localement.

Noting the steady increase in Chinese tourism to Thailand, this article examines the emergence of a new category of tourism stakeholders, on the island of Phuket in particular. A series of entrepreneurs have settled there to open "pre-wedding" photography workshops, the halfway practice between a honeymoon and a themed stay. This article aims to understand this phenomenon, while opening the discussion on the creation of an economic system that supports it locally. 
INDEX

Mots-clés : Thaillande, photos de mariage, tourisme international chinois, adaptation, acteurs touristiques

Keywords : Thailand, wedding photos, Chinese international tourism, adaptation, tourism stakeholders

\section{AUTEUR}

\section{MENG LI}

PhD

Humanities \& Social Sciences Department

College of Arts and Sciences

Gulf University of Sciences and Technology

Li.M[at]gust.edu.kw 